\title{
Prevalence of Malnutrition and Associated Risk Factors Among Children Under Five Years of age in Amhara Region, Ethiopia: Evidence from 2016 Ethiopian Demographic and Health Survey
}

Damitie Kebede Mengesha ( $\nabla$ dakebede10@gmail.com )

Bahir Dar University https://orcid.org/0000-0001-5494-9906

Yidnekachew Merkeb

Bahir Dar University

\section{Research}

Keywords: Malnutrition, Young children, Underweight, Wasting, Stunting, Ethiopia

Posted Date: August 10th, 2020

DOI: https://doi.org/10.21203/rs.3.rs-54072/v1

License: (a) (1) This work is licensed under a Creative Commons Attribution 4.0 International License.

Read Full License 


\section{Abstract}

Background: Under-nutrition is the outcome of insufficient food intake and recurrent infectious diseases. Childhood malnutrition is most widely prevalent among children under the age of five in Amhara Region, Ethiopia. This study explored the major determinants of malnutrition and its association with anemia among children under five years of age in Amhara Region, Ethiopia.

Methods: Data from the 2016 Ethiopian Demographic and Health Survey (EDHS) was used. A total of 974 children under five years of age were used. A multivariable binary logistic regression analysis was used at a $5 \%$ level of significance to determine the individual- and community-level factors associated with childhood malnutrition.

Results: The prevalence of stunting, wasting, and underweight were $46.3 \%, 9.8 \%$, and $28.4 \%$, respectively. About $23.1 \%$ of children were both stunting and underweight, $7.3 \%$ were both underweight and wasting, and $4.5 \%$ of children had all the three conditions. Among the factors considered in this study, Age of child in months, birth weight of the child at birth, mother's highest education level, sex of household head, sources of drinking water, and type of toilet facility were significantly associated with malnutrition in Amhara Region.

Conclusion: Malnutrition among under-five children was one of the public health problems in the Amhara Region. The influence of these factors should be considered to develop strategies for reducing malnutrition in Amhara Region. Finally improving the living standards of the children is important to get better health care, to enhance the child's nutritional status, and reduce child mortality.

\section{Background}

Malnutrition remains a critical public health problem among children under the age of five years in developing countries including Ethiopia. Malnutrition is caused by multiple interlinked factors and has both short and long term detrimental health effects [1]. It affects the cognitive and physical development of children, raises the risk of infections, and significantly contributes to child morbidity and mortality [2]. Stunting, being underweight and wasting are three widely recognized indicators of a child's nutritional status. Whereas stunting and wasting direct chronic and acute malnutrition respectively, being underweight is a composite pointer and includes both acute and chronic malnutrition. However, different forms of malnutrition can also occur concurrently in children [3].

In the Amhara region, the prevalence of stunting declined from $56.6 \%$ in 2005 to $52 \%$ in 2011 ; the prevalence of wasting decreased from $14.2 \%$ in 2005 to $9.9 \%$ in 2011 ; the prevalence of being underweight sharply decreased from $48.9 \%$ in 2005 to $33.4 \%$ in 2011 . The prevalence of stunting declined from $58 \%$ in 2000 to $44 \%$ in 2011 in Ethiopia. The prevalence of wasting changed from $12 \%$ in 2000 to $10 \%$ in 2011 . The prevalence of being underweight steadily decreased from $41 \%$ in 2000 to $29 \%$ in 2011[4]. In Tanzania, a high prevalence of being underweight (46.0\%), stunting (41.9\%) and wasting (24.7\%) was observed in 2017. Additionally, 33\% of children have shared stunting and underweight, $21 \%$ 
of children were both underweight and wasting, and $12 \%$ of children were stunted and wasting [5]. In Ethiopia, more than one-third of child deaths are associated with malnutrition [6].

Although studies have been carried out on the identification of factors that are associated with children under five years old of malnutrition in the Amhara region, none of them uses the nationally representative data for the Amhara region. The effort made in decreasing under-five children's malnutrition in the region is still high, and more effort is needed to improve the barriers for further reduction. More research studies are, therefore, required to inform policymakers to implement appropriate intervention programs. To address these gaps, an all-inclusive cross-sectional analysis of the recent 2016 Ethiopian Demographic Health Survey (EDHS) was done, to explore the major risk factors of malnutrition and its association with anemia among under-five children in Amhara Region, Ethiopia.

\section{Methods}

\section{Description of study design and area}

Cross-sectional study design was used for this study. The study was conducted in Amhara regional state which is located in the north western and north central part of Ethiopia. According to the 2007 Census, the state's population birth weight was $17,214,056$ of which $8,636,1875$ were males. The urban residents of the region were 2,112,220 and its rural residents 15,101,836 [7].

\section{Inclusion And Exclusion Variables}

Mothers/caregivers who had young children under five years old who live in the Amhara regional state for at least 6 months were included in the study and those who had mental illnesses interfering with the interview were not considered in the study.

\section{Sources Of Data}

The data onto this study were extracted from the EDHS 2016. The 2016 EDHS used a two-stage stratified sampling to select households. In the first stage, there were 645 enumeration areas (202 in urban and 443 in rural areas) based on the 2007 Ethiopia Population and Housing Census (PHC). In the second stage, A total of 18,008 households were considered, of which 16,650 (98\% of response rate) households were eligible. The women were interviewed by distributing questioners and information on their birth history [4]. About 974 (501 males and 473 females) under-five children from Amhara region were considered for this study.

\section{Study Variables}


The dependent variables for this study are the malnutrition status of under-5 year children (stunting, underweight and wasting). A child whose height for age Z-score is below minus two standard deviations $(-2 S D)$ from the median of the reference population is considered as stunting. If the weight for age Zscore is below minus two standard deviations (-2SD) from the median of the reference population then the child is underweight. Children whose weight for height Z-score is below minus two standard deviations (-2SD) from the median of the reference population are considered as wasting [8].

Determinants of stunting, wasting, and underweight in this study was selected from the available similar studies on the subject [8-13]. The risk factors associated included socio-demographic, maternal and child factors. Socio demographic-maternal factors selected were types of residence, household wealth index, mother's educational level, mother's body mass index (BMI), religion, type of toilet facility, sex of household head and sources of drinking water. Child-level factors were the sex of the child, child age, type of birth, number of living children and childbirth weight at birth.

\section{Statistical Data Analysis}

The data was extracted, edited, and analyzed by using SPSS version 23 for Windows. The descriptive statistics such as frequencies and proportions were used to summarize the distribution of selected background characteristics of the sample. Bivariate logistic regression was performed and a variable with a P-value of less than 0.25 was transported into a multivariable binary logistic regression analysis to identify the determinants of malnutrition of children under five years old. Finally, variables with P-values < 0.05 in the multivariable logistic regression model were taken as statistically significant.

\section{Results}

Our population included 974 children under the age of five years from the Amhara region were considered in this research. The prevalence of stunting, being underweight, and wasting in Amhara region were $46.3 \%, 28.4 \%$, and $9.8 \%$, respectively. About $23.1 \%$ of children were both stunted and underweight, $7.3 \%$ were both underweight and wasting, and $4.5 \%$ of children had all the three conditions.

Of that $23.3 \%$ were $>4 \mathrm{~kg}$ birth weight and only $2.9 \%$ were multiple birth types. About $74.9 \%$ of interviewed mothers had no education and only $2.1 \%$ of them attended higher education. About $42.6 \%$ of children were found between 0 to 24 months and more than half $(51.3 \%)$ were males. Only $9.1 \%$ of the respondents were from urban areas and $30.2 \%$ were in the rich wealth index. Around $19.8 \%$ of children's mothers were underweight.

\section{Determinants Of Stunting}

Among the factors considered in this study, Age of child in months, birth weight of the child at birth, mother highest education level, sex of household head and sources of drinking water were associated 
with stunting. The log odds of stunting was higher among children in the age group of 25-47 months (Adjusted Odds Ratio $(\mathrm{AOR})=1.57,95 \% \mathrm{Cl}: 1.05-2.35)$ and $48-59$ months $(\mathrm{AOR}=1.06,95 \% \mathrm{Cl}: 70.80-$ 1.49) respectively as compared to the age group of $0-24$ months. Compared to children $>4 \mathrm{~kg}$ birth weight at birth, the odds of stunting among children in the $2.5-4 \mathrm{~kg}$ birth weight was 0.01 times lower. The odd of stunting among children in the $<2.5 \mathrm{~kg}$ birth weight were 1.60 times higher compared to children's $>4 \mathrm{~kg}$ birth weight at birth.

The risk of stunting among children whose mothers attended primary education was 1.07 ( $A O R=1.07$, $95 \% \mathrm{Cl}: 0.76-1.50)$ times more compared to children whose mothers did not attend education. The risk of stunting among children whose mothers attended secondary education was 0.70 times less compared to children whose mothers did not attend education. The risk of stunting among children whose father household head was 0.49 times less compared to children whose mother household headed. Children from households that used unimproved drinking water were $1.47(\mathrm{AOR}=1.47,95 \% \mathrm{Cl}: 1.11-1.95)$ times more likely to be at risk of stunting than children from households that used improved water. 
Table 1

Bivariate and multivariable logistic regression of risk factors associated with stunting on childhood less than 5 years old in Amhara Region, Ethiopia, EDHS 2016.

\begin{tabular}{|c|c|c|c|c|}
\hline \multirow[t]{2}{*}{ Variables } & & Stunting & COR (95\% Cl) & AOR $(95 \% \mathrm{Cl})$ \\
\hline & Yes & \multicolumn{3}{|l|}{ No } \\
\hline \multicolumn{5}{|c|}{ Age of child in months } \\
\hline $0-24$ & $\begin{array}{l}169 \\
(43.7 \%)\end{array}$ & $\begin{array}{l}218 \\
(56.3 \%)\end{array}$ & 1 & 1 \\
\hline $25-47$ & $\begin{array}{l}149 \\
(45.8 \%)\end{array}$ & $\begin{array}{l}176 \\
(54.2 \%)\end{array}$ & $\begin{array}{l}1.09(0.81, \\
1.47)\end{array}$ & $1.57(1.05,2.35)^{\star}$ \\
\hline $48-59$ & $\begin{array}{l}102 \\
(51.5 \%)\end{array}$ & $96(48.5 \%)$ & $\begin{array}{l}1.37(0.97 \\
1.93)\end{array}$ & $1.06(0.80,1.49)$ \\
\hline \multicolumn{5}{|l|}{ Sex of child } \\
\hline Male & $\begin{array}{l}218 \\
(43.6 \%)\end{array}$ & $\begin{array}{l}282 \\
(56.4 \%)\end{array}$ & 1 & 1 \\
\hline Female & $\begin{array}{l}232 \\
(48.9 \%)\end{array}$ & $\begin{array}{l}242 \\
(51.1 \%)\end{array}$ & $\begin{array}{l}1.24(0.96 \\
1.60)\end{array}$ & $1.27(0.97,1.67)$ \\
\hline \multicolumn{5}{|c|}{ Place of residence } \\
\hline Urban & $39(43.8 \%)$ & $50(56.2 \%)$ & 1 & 1 \\
\hline Rural & $\begin{array}{l}411 \\
(46.4 \%)\end{array}$ & $\begin{array}{l}474 \\
(53.6 \%)\end{array}$ & $\begin{array}{l}1.11(0.72, \\
1.72)\end{array}$ & $0.53(.26,1.06)$ \\
\hline \multicolumn{5}{|l|}{ Religion } \\
\hline Orthodox & $\begin{array}{l}380 \\
(46.3 \%)\end{array}$ & $\begin{array}{l}441 \\
(53.7 \%)\end{array}$ & 1 & 1 \\
\hline Muslin & $70(46.1 \%)$ & $82(53.9 \%)$ & $\begin{array}{l}0.99(0.70 \\
1.40)\end{array}$ & $1.07(0.69,1.66)$ \\
\hline \multicolumn{5}{|l|}{ Type of birth } \\
\hline Single birth & $\begin{array}{l}438 \\
(46.3 \%)\end{array}$ & $\begin{array}{l}508 \\
(53.7 \%)\end{array}$ & 1 & 1 \\
\hline Multiple birth & $12(42.9 \%)$ & $16(57.1 \%)$ & $\begin{array}{l}0.87(0.41 \\
1.86)\end{array}$ & $1.18(0.41,3.39)$ \\
\hline \multicolumn{5}{|l|}{ Mothers` BMI } \\
\hline Over weight & $15(41.7 \%)$ & $21(58.3 \%)$ & 1 & 1 \\
\hline
\end{tabular}

AOR: adjusted odds ratio; COR: crude odds ratio; $\mathrm{Cl}$ : confidence interval; * significant $\mathrm{p}$-value $<0.05$; ** significant $p$-value $<0.01 ; 1=$ reference 


\begin{tabular}{|c|c|c|c|c|}
\hline \multicolumn{2}{|l|}{ Variables } & \multirow{2}{*}{$\begin{array}{l}\text { Stunting } \\
387 \\
(52.4 \%)\end{array}$} & \multirow{2}{*}{$\begin{array}{l}\text { COR }(95 \% \mathrm{Cl}) \\
1.01(0.49 \\
2.06)\end{array}$} & \multirow{2}{*}{$\begin{array}{l}\text { AOR }(95 \% \mathrm{Cl}) \\
0.95(0.37,2.43)\end{array}$} \\
\hline Normal weight & $\begin{array}{l}351 \\
(47.6 \%)\end{array}$ & & & \\
\hline Under weight & $81(41.8 \%)$ & $\begin{array}{l}113 \\
(58.2 \%)\end{array}$ & $\begin{array}{l}1.40(0.30 \\
6.51)\end{array}$ & $0.78(0.10,6.21)$ \\
\hline \multicolumn{5}{|c|}{ Birth weight of the child at birth } \\
\hline$>4 \mathrm{~kg}$ & $99(43.6 \%)$ & $\begin{array}{l}128 \\
(56.4 \%)\end{array}$ & 1 & 1 \\
\hline $2.5-4$ kg & $\begin{array}{l}176 \\
(42.1 \%)\end{array}$ & $\begin{array}{l}242 \\
(57.9 \%)\end{array}$ & $\begin{array}{l}0.94(0.68 \\
1.30)\end{array}$ & $0.99(0.70,1.42)$ \\
\hline$<2.5 \mathrm{~kg}$ & $\begin{array}{l}175 \\
(53.2 \%)\end{array}$ & $\begin{array}{l}154 \\
(46.8 \%)\end{array}$ & $\begin{array}{l}1.47(1.05 \\
2.07)\end{array}$ & $\begin{array}{l}1.60(1.11 \\
2.31)^{\star \star}\end{array}$ \\
\hline \multicolumn{5}{|c|}{ Mother highest educational level } \\
\hline No education & $\begin{array}{l}343 \\
(46.9 \%)\end{array}$ & $\begin{array}{l}388 \\
(53.1 \%)\end{array}$ & 1 & 1 \\
\hline Primary & $87(47.3 \%)$ & $97(52.7 \%)$ & $\begin{array}{l}1.02(0.72 \\
1.40)\end{array}$ & $1.07(0.76,1.50)$ \\
\hline Secondary & $7(18.4 \%)$ & $31(81.6 \%)$ & $\begin{array}{l}0.26(0.11 \\
0.59)\end{array}$ & $0.30(0.13,0.70)^{\star}$ \\
\hline Higher & $7(18.4 \%)$ & $31(81.6 \%)$ & $\begin{array}{l}1.84(0.75 \\
4.49)\end{array}$ & $2.37(0.87,6.52)$ \\
\hline \multicolumn{5}{|c|}{ Type of toilet facility } \\
\hline Improved & $\begin{array}{l}236 \\
(48.0 \%)\end{array}$ & $\begin{array}{l}256 \\
(52.0 \%)\end{array}$ & 1 & 1 \\
\hline Unimproved & $\begin{array}{l}215 \\
(44.6 \%)\end{array}$ & $\begin{array}{l}267 \\
(55.4 \%)\end{array}$ & $\begin{array}{l}1.15(0.89 \\
1.47)\end{array}$ & $1.30(0.96,1.77)$ \\
\hline \multicolumn{5}{|c|}{ Sex of household head } \\
\hline Female & $36(40.4 \%)$ & $53(59.6 \%)$ & 1 & 1 \\
\hline Male & $\begin{array}{l}414 \\
(46.8 \%)\end{array}$ & $\begin{array}{l}471 \\
(53.2 \%)\end{array}$ & $\begin{array}{l}1.29 \\
2.02)\end{array}$ & $0.51(0.28,0.91)^{*}$ \\
\hline \multicolumn{5}{|c|}{$\begin{array}{l}\text { Household wealth index } \\
\text { combined }\end{array}$} \\
\hline Poor & $\begin{array}{l}210 \\
(46.7 \%)\end{array}$ & $\begin{array}{l}240 \\
(53.3 \%)\end{array}$ & 1 & 1 \\
\hline
\end{tabular}

AOR: adjusted odds ratio; COR: crude odds ratio; $\mathrm{Cl}$ : confidence interval; * significant $\mathrm{p}$-value $<0.05$; ** significant $p$-value $<0.01 ; 1=$ reference 


\begin{tabular}{|c|c|c|c|c|}
\hline Variables & & Stunting & $\operatorname{COR}(95 \% \mathrm{Cl})$ & AOR (95\% Cl) \\
\hline Medium & $\begin{array}{l}109 \\
(47.4 \%)\end{array}$ & $\begin{array}{l}121 \\
(52.6 \%)\end{array}$ & $\begin{array}{l}1.03(0.75 \\
1.42)\end{array}$ & $1.04(0.71,1.52)$ \\
\hline Rich & $\begin{array}{l}131 \\
(44.6 \%)\end{array}$ & $\begin{array}{l}163 \\
(55.4 \%)\end{array}$ & $\begin{array}{l}0.92(0.68, \\
1.23)\end{array}$ & $0.77(0.52,1.14)$ \\
\hline \multicolumn{5}{|c|}{ Number of living children } \\
\hline $1-2$ & $\begin{array}{l}160 \\
(45.8 \%)\end{array}$ & $\begin{array}{l}189 \\
(54.2 \%)\end{array}$ & 1 & 1 \\
\hline $3-4$ & $80(46.8 \%)$ & $91(53.2 \%)$ & $\begin{array}{l}0.99(0.75 \\
1.31)\end{array}$ & $1.08(0.77,1.52)$ \\
\hline$>4$ & $\begin{array}{l}207 \\
(46.1 \%)\end{array}$ & $\begin{array}{l}242 \\
(53.9 \%)\end{array}$ & $\begin{array}{l}1.03(0.72, \\
1.46)\end{array}$ & $1.26(0.82,1.94)$ \\
\hline \multicolumn{5}{|c|}{ Sources of drinking water } \\
\hline Improved & $\begin{array}{l}178 \\
(50.9 \%)\end{array}$ & $\begin{array}{l}172 \\
(49.1 \%)\end{array}$ & 1 & 1 \\
\hline Unimproved & $\begin{array}{l}273 \\
(43.8 \%)\end{array}$ & $\begin{array}{l}351 \\
(56.2 \%)\end{array}$ & $\begin{array}{l}0.75(0.58 \\
0.98)\end{array}$ & $\begin{array}{l}1.47(1.11 \\
1.95)^{\star \star}\end{array}$ \\
\hline
\end{tabular}

\section{Determinants Of Under-weight}

The birth weight of the child at birth was associated with under-weight $(P<0.05)$. The risk of being underweight was 0.22 times less likely among children that were aged 25-47 months than those aged 0-24 months. The risk of being underweight was 1.36 times more likely among children that were aged 48-59 months than those aged 0-24 months. The risk of being underweight for children whose mother attended primary and secondary education were 0.23 and 0.35 times lower than children whose mothers who did not attend formal education respectively.

Children from a household with rich economic status were 0.09 times less likely to be under-weighted compared to children living in a household with poor household economic status. Children from rural areas were 1.16 times more likely to be underweight compared to children from urban areas. Children who were born with $<2.5 \mathrm{~kg}$ birth weight were 1.80 times more likely to be underweight than children born $>4 \mathrm{~kg}$ birth weight $(\mathrm{AOR}=1.80 ; 95 \% \mathrm{CL} 0.89-3.66)$ and children who had born with 2.5-4 kg birth weight were 1.56 times more likely to be under-weighted than children born $>4 \mathrm{~kg}$ birth weight $(\mathrm{AOR}=1.56 ; 95 \%$ CL 1.05-2.33). 
Table 2

Bivariate and multivariable logistic regression of risk factors associated with under-weight on childhood less than 5 years in Amhara Region, Ethiopia, EDHS 2016

\begin{tabular}{|c|c|c|c|c|}
\hline \multirow[t]{2}{*}{ Variables } & & Underweight & COR $(95 \% \mathrm{Cl})$ & AOR $(95 \% \mathrm{Cl})$ \\
\hline & Yes & \multicolumn{3}{|l|}{ No } \\
\hline \multicolumn{5}{|c|}{ Age of child in months } \\
\hline $0-24$ & $\begin{array}{l}104 \\
(26.9 \%)\end{array}$ & $283(73.1 \%)$ & 1 & 1 \\
\hline $25-47$ & $86(26.5 \%)$ & $239(73.5 \%)$ & $0.98(0.70,1.37)$ & $\begin{array}{l}0.78(0.53, \\
1.16)\end{array}$ \\
\hline $48-59$ & $63(31.8 \%)$ & $135(68.2 \%)$ & $1.27(0.87,1.85)$ & $\begin{array}{l}1.36(0.89 \\
2.09)\end{array}$ \\
\hline \multicolumn{5}{|l|}{ Sex of child } \\
\hline Male & $\begin{array}{l}134 \\
(26.8 \%)\end{array}$ & $366(73.2 \%)$ & 1 & 1 \\
\hline Female & $\begin{array}{l}142 \\
(30.0 \%)\end{array}$ & $332(70.0 \%)$ & $1.17(0.88,1.54)$ & $\begin{array}{l}1.06(0.76 \\
1.49)\end{array}$ \\
\hline \multicolumn{5}{|c|}{ Place of residence } \\
\hline Urban & $15(16.9 \%)$ & $74(83.1 \%)$ & 1 & 1 \\
\hline Rural & $\begin{array}{l}261 \\
(29.5 \%)\end{array}$ & $624(70.5 \%)$ & $2.06(1.16,3.66)$ & $\begin{array}{l}1.16(0.50 \\
2.69)\end{array}$ \\
\hline \multicolumn{5}{|l|}{ Religion } \\
\hline Orthodox & $\begin{array}{l}228 \\
(27.8 \%)\end{array}$ & $593(72.2 \%)$ & 1 & 1 \\
\hline Muslin & $48(31.6 \%)$ & $104(68.4 \%)$ & $1.20(0.83,1.75)$ & $\begin{array}{l}1.60(1.01 \\
2.54)\end{array}$ \\
\hline \multicolumn{5}{|l|}{ Type of birth } \\
\hline Single birth & $\begin{array}{l}268 \\
(28.3 \%)\end{array}$ & $678(71.7 \%)$ & 1 & 1 \\
\hline Multiple birth & $8(28.6 \%)$ & $20(71.4 \%)$ & $1.01(0.44,2.32)$ & $\begin{array}{l}1.32(0.43 \\
4.03)\end{array}$ \\
\hline \multicolumn{5}{|l|}{ Mothers` BMI } \\
\hline Over weight & 8 (22.2\%) & $28(77.8 \%)$ & 1 & 1 \\
\hline
\end{tabular}

AOR: adjusted odds ratio; COR: crude odds ratio; Cl: confidence interval; * significant p-value $<0.05$; 1 $=$ reference 


\begin{tabular}{|c|c|c|c|c|}
\hline Variables & & Underweight & COR $(95 \% \mathrm{Cl})$ & AOR $(95 \% \mathrm{Cl})$ \\
\hline Normal weight & $\begin{array}{l}209 \\
(28.3 \%)\end{array}$ & $529(71.7 \%)$ & $1.46(0.63,3.39)$ & $\begin{array}{l}2.28(0.63 \\
8.27)\end{array}$ \\
\hline Under weight & $57(29.4 \%)$ & $137(70.6 \%)$ & $1.17(0.20,6.94)$ & $\begin{array}{l}0.88(0.07 \\
11.51)\end{array}$ \\
\hline \multicolumn{5}{|c|}{ Birth weight of the child at birth } \\
\hline$>4 \mathrm{~kg}$ & $61(26.9 \%)$ & $166(73.1 \%)$ & 1 & 1 \\
\hline $2.5-4 \mathrm{~kg}$ & $\begin{array}{l}101 \\
(24.2 \%)\end{array}$ & $317(75.8 \%)$ & $0.87(0.60,1.25)$ & $\begin{array}{l}1.56(1.05 \\
2.33)^{\star}\end{array}$ \\
\hline$<2.5 \mathrm{~kg}$ & $\begin{array}{l}114 \\
(34.7 \%)\end{array}$ & $215(65.3 \%)$ & $1.44(0.99,2.09)$ & $\begin{array}{l}1.80(0.89 \\
3.66)\end{array}$ \\
\hline \multicolumn{5}{|c|}{$\begin{array}{l}\text { Mother highest educational } \\
\text { level }\end{array}$} \\
\hline No education & $\begin{array}{l}217 \\
(29.7 \%)\end{array}$ & $514(70.3 \%)$ & 1 & 1 \\
\hline Primary & $47(25.5 \%)$ & $137(74.5 \%)$ & $0.82(0.56,1.17)$ & $\begin{array}{l}0.89(0.58 \\
1.38)\end{array}$ \\
\hline Secondary & $5(13.2 \%)$ & $33(86.8 \%)$ & $0.36(0.14,0.93)$ & $\begin{array}{l}0.25(0.05 \\
1.14)\end{array}$ \\
\hline Higher & $7(33.3 \%)$ & $14(66.7 \%)$ & $1.18(0.47,2.96)$ & $\begin{array}{l}1.92(0.50 \\
7.34)\end{array}$ \\
\hline \multicolumn{5}{|c|}{ Type of toilet facility } \\
\hline Improved & $\begin{array}{l}130 \\
(26.4 \%)\end{array}$ & $362(73.6 \%)$ & 1 & 1 \\
\hline Unimproved & $\begin{array}{l}146 \\
(30.3 \%)\end{array}$ & $336(69.7 \%)$ & $0.83(0.62,1.09)$ & $\begin{array}{l}0.80(0.57 \\
1.12)\end{array}$ \\
\hline \multicolumn{5}{|c|}{ Sex of household head } \\
\hline Female & $25(28.1 \%)$ & $64(71.9 \%)$ & 1 & 1 \\
\hline Male & $\begin{array}{l}251 \\
(28.4 \%)\end{array}$ & $634(71.6 \%)$ & $0.99(0.61,1.60)$ & $\begin{array}{l}0.53(0.27 \\
1.05)\end{array}$ \\
\hline \multicolumn{5}{|c|}{$\begin{array}{l}\text { Household wealth index } \\
\text { combined }\end{array}$} \\
\hline Poor & $\begin{array}{l}133 \\
(29.6 \%)\end{array}$ & $317(70.4 \%)$ & 1 & 1 \\
\hline
\end{tabular}

AOR: adjusted odds ratio; COR: crude odds ratio; Cl: confidence interval; * significant $p$-value < 0.05; 1 $=$ reference 


\begin{tabular}{|c|c|c|c|c|}
\hline Variables & & Underweight & COR $(95 \% \mathrm{Cl})$ & AOR $(95 \% \mathrm{Cl})$ \\
\hline Medium & $67(29.1 \%)$ & $163(70.9 \%)$ & $0.98(0.69,1.39)$ & $\begin{array}{l}1.07(0.71 \\
1.61)\end{array}$ \\
\hline Rich & $76(25.9 \%)$ & $218(74.1 \%)$ & $0.83(0.60,1.16)$ & $\begin{array}{l}0.91(0.59 \\
1.40)\end{array}$ \\
\hline \multicolumn{5}{|c|}{ Number of living children } \\
\hline $1-2$ & $85(24.4 \%)$ & $264(75.6 \%)$ & 1 & 1 \\
\hline $3-4$ & $54(31.6 \%)$ & $117(68.4 \%)$ & $0.75(0.55,1.03)$ & $\begin{array}{l}0.86(0.59 \\
1.26)\end{array}$ \\
\hline$>4$ & $\begin{array}{l}135 \\
(30.1 \%)\end{array}$ & $314(69.9 \%)$ & $\begin{array}{l}0.1 .07(0.73, \\
3.90)\end{array}$ & $\begin{array}{l}1.25(0.79 \\
1.97)\end{array}$ \\
\hline \multicolumn{5}{|c|}{ Sources of drinking water } \\
\hline Improved & $\begin{array}{l}113 \\
(32.3 \%)\end{array}$ & $237(67.7 \%)$ & 1 & 1 \\
\hline Unimproved & $\begin{array}{l}163 \\
(26.1 \%)\end{array}$ & $461(73.9 \%)$ & $1.35(1.01,1.80)$ & $\begin{array}{l}1.20(0.85 \\
1.69)\end{array}$ \\
\hline \multicolumn{5}{|c|}{$\begin{array}{l}\text { AOR: adjusted odds ratio; COR: crude odds ratio; Cl: confidence interval; * significant } p \text {-value }<0.05 ; 1 \\
=\text { reference }\end{array}$} \\
\hline
\end{tabular}

\section{Determinants Of Wasting}

Results of the multivariable binary logistic regression model showed that the type of toilet facility and sex of household head were significantly associated with wasting. Children living in a household with improved toilet type were 0.48 less likely to be wasting compared to children living in a household with unimproved toilet type. Children from a male household head were 1.99 times higher compared to children from a female household head. Children of the rich household were 0.32 times less likely to be wasting compared to children living in a household with poor household economic status.

The risk of wasting was 1.08 and 1.52 times higher among children of 25-47 and 48-59 months than those 0-24 months, respectively. The odds of wasting of children from rural areas were 1.18 times higher compared to children from urban areas. The odds of wasting were 0.04 times lower among female children than male children. The odds of wasting was 1.17 times higher among children who lived in household members of $>4$ children who had lived in household members of $1-2(\mathrm{AOR}=1.17,95 \% \mathrm{Cl}$ $0.59-2.33)$. 
Table 3

Bivariate and multivariable logistic regression of risk factors associated with wasting on childhood less than 5 years in Amhara Region, Ethiopia, EDHS 2016

\begin{tabular}{|c|c|c|c|c|}
\hline \multirow[t]{2}{*}{ Variables } & & Wasting & $\operatorname{COR}(95 \% \mathrm{Cl})$ & AOR (95\% Cl) \\
\hline & Yes & \multicolumn{3}{|l|}{ No } \\
\hline \multicolumn{5}{|c|}{ Age of child in months } \\
\hline $0-24$ & $33(8.5 \%)$ & $\begin{array}{l}354 \\
(91.5 \%)\end{array}$ & 1 & 1 \\
\hline $25-47$ & $30(9.2 \%)$ & $\begin{array}{l}295 \\
(90.8 \%)\end{array}$ & $\begin{array}{l}1.09(0.65 \\
1.83)\end{array}$ & $1.08(0.64,1.82)$ \\
\hline $48-59$ & $\begin{array}{l}25 \\
(12.6 \%)\end{array}$ & $\begin{array}{l}173 \\
(87.4 \%)\end{array}$ & $\begin{array}{l}1.55(0.89 \\
2.69)\end{array}$ & $1.52(0.87,2.64)$ \\
\hline \multicolumn{5}{|l|}{ Sex of child } \\
\hline Male & $\begin{array}{l}50 \\
(10.0 \%)\end{array}$ & $\begin{array}{l}450 \\
(90.0 \%)\end{array}$ & 1 & 1 \\
\hline Female & $46(9.7 \%)$ & $\begin{array}{l}428 \\
(90.3 \%)\end{array}$ & $\begin{array}{l}0.97(0.63 \\
1.48)\end{array}$ & $0.96(0.58,1.58)$ \\
\hline \multicolumn{5}{|c|}{ Place of residence } \\
\hline Urban & $7(7.9 \%)$ & $82(92.1 \%)$ & 1 & 1 \\
\hline Rural & $\begin{array}{l}89 \\
(10.1 \%)\end{array}$ & $\begin{array}{l}796 \\
(89.9 \%)\end{array}$ & $\begin{array}{l}1.31(0.59 \\
2.92)\end{array}$ & $1.18(0.36,3.88)$ \\
\hline \multicolumn{5}{|l|}{ Religion } \\
\hline Orthodox & $79(9.6 \%)$ & $\begin{array}{l}742 \\
(90.4 \%)\end{array}$ & 1 & 1 \\
\hline Muslin & $\begin{array}{l}17 \\
(11.2 \%)\end{array}$ & $\begin{array}{l}135 \\
(88.8 \%)\end{array}$ & $\begin{array}{l}1.18(0.68, \\
2.06)\end{array}$ & $1.22(0.62,2.37)$ \\
\hline \multicolumn{5}{|l|}{ Type of birth } \\
\hline Single birth & $94(9.9 \%)$ & $\begin{array}{l}852 \\
(90.1 \%)\end{array}$ & 1 & 1 \\
\hline Multiple birth & $2(7.1 \%)$ & $26(92.9 \%)$ & $\begin{array}{l}0.68(0.16 \\
2.98)\end{array}$ & $0.53(0.06,4.32)$ \\
\hline \multicolumn{5}{|l|}{ Mothers` BMI } \\
\hline Over weight & 5 (13.9\%) & 31 (86.1\%) & 1 & 1 \\
\hline
\end{tabular}

AOR: adjusted odds ratio; COR: crude odds ratio; Cl: confidence interval; * significant $p$-value $<0.05$; ** significant $p$-value $<0.01 ; 1=$ reference 


\begin{tabular}{|c|c|c|c|c|}
\hline \multicolumn{2}{|l|}{ Variables } & \multirow{2}{*}{$\begin{array}{l}\text { Wasting } \\
\begin{array}{l}667 \\
(90.4 \%)\end{array}\end{array}$} & \multirow{2}{*}{$\begin{array}{l}\text { COR }(95 \% \text { Cl }) \\
0.67(0.23 \\
1.94)\end{array}$} & \multirow{2}{*}{$\begin{array}{l}\text { AOR }(95 \% \mathrm{Cl}) \\
2.50(0.32,19.44)\end{array}$} \\
\hline Normal weight & $71(9.6 \%)$ & & & \\
\hline Under weight & $19(9.8 \%)$ & $\begin{array}{l}175 \\
(90.2 \%)\end{array}$ & $\begin{array}{l}0.89(0.09 \\
8.82)\end{array}$ & $2.73(0.34,22.08)$ \\
\hline \multicolumn{5}{|c|}{ Birth weight of the child at birth } \\
\hline$>4 \mathrm{~kg}$ & $22(9.7 \%)$ & $\begin{array}{l}205 \\
(90.3 \%)\end{array}$ & 1 & 1 \\
\hline $2.5-4 \mathrm{~kg}$ & $\begin{array}{l}46 \\
(11.0 \%)\end{array}$ & $\begin{array}{l}372 \\
(89.0 \%)\end{array}$ & $\begin{array}{l}1.15(0.67 \\
1.97)\end{array}$ & $1.36(0.71,2.61)$ \\
\hline$<2.5 \mathrm{~kg}$ & $28(8.5 \%)$ & $\begin{array}{l}301 \\
(91.5 \%)\end{array}$ & $\begin{array}{l}0.87(0.48 \\
1.56)\end{array}$ & $0.87(0.42,1.77)$ \\
\hline \multicolumn{5}{|c|}{ Mother highest educational level } \\
\hline No education & $72(9.8 \%)$ & $\begin{array}{l}659 \\
(90.2 \%)\end{array}$ & 1 & 1 \\
\hline Primary & $18(9.8 \%)$ & $\begin{array}{l}166 \\
(90.2 \%)\end{array}$ & $\begin{array}{l}0.99(0.58 \\
1.71)\end{array}$ & $1.32(0.71,2.46)$ \\
\hline Secondary & $4(10.5 \%)$ & $34(89.5 \%)$ & $\begin{array}{l}1.08(0.37 \\
3.12)\end{array}$ & $1.63(0.39,6.73)$ \\
\hline Higher & $2(9.5 \%)$ & $19(90.5 \%)$ & $\begin{array}{l}0.96(0.22 \\
4.22)\end{array}$ & $2.96(0.46,19.07)$ \\
\hline \multicolumn{5}{|c|}{ Type of toilet facility } \\
\hline Improved & $39(7.9 \%)$ & $\begin{array}{l}453 \\
(92.1 \%)\end{array}$ & 1 & 1 \\
\hline Unimproved & $\begin{array}{l}57 \\
(11.8 \%)\end{array}$ & $\begin{array}{l}425 \\
(88.2 \%)\end{array}$ & $\begin{array}{l}0.64(0.42 \\
0.99)\end{array}$ & $\begin{array}{l}0.52(0.31 \\
0.87)^{\star \star}\end{array}$ \\
\hline \multicolumn{5}{|c|}{ Sex of household head } \\
\hline Female & $\begin{array}{l}14 \\
(15.7 \%)\end{array}$ & $75(84.3 \%)$ & 1 & 1 \\
\hline Male & $82(9.3 \%)$ & $\begin{array}{l}803 \\
(90.7 \%)\end{array}$ & $\begin{array}{l}1.83(0.99 \\
3.39)\end{array}$ & $1.99(1.06,3.73)$ * \\
\hline \multicolumn{5}{|c|}{$\begin{array}{l}\text { Household wealth index } \\
\text { combined }\end{array}$} \\
\hline Poor & $\begin{array}{l}46 \\
(10.2 \%)\end{array}$ & $\begin{array}{l}404 \\
(89.8 \%)\end{array}$ & 1 & 1 \\
\hline
\end{tabular}

AOR: adjusted odds ratio; COR: crude odds ratio; Cl: confidence interval; * significant $\mathrm{p}$-value < 0.05 ; ** significant $p$-value $<0.01 ; 1=$ reference 


\begin{tabular}{|c|c|c|c|c|}
\hline Variables & & Wasting & COR $(95 \% \mathrm{Cl})$ & AOR (95\% Cl) \\
\hline Medium & $\begin{array}{l}29 \\
(12.6 \%)\end{array}$ & $\begin{array}{l}201 \\
(87.4 \%)\end{array}$ & $\begin{array}{l}1.27(0.77 \\
2.08)\end{array}$ & $1.12(0.66,1.90)$ \\
\hline Rich & $21(7.1 \%)$ & $\begin{array}{l}273 \\
(92.9 \%)\end{array}$ & $\begin{array}{l}0.68(0.39 \\
1.16)\end{array}$ & $0.68(0.38,1.19)$ \\
\hline \multicolumn{5}{|c|}{ Number of living children } \\
\hline $1-2$ & $30(8.6 \%)$ & $\begin{array}{l}319 \\
(91.4 \%)\end{array}$ & 1 & 1 \\
\hline $3-4$ & $17(9.9 \%)$ & $\begin{array}{l}154 \\
(90.1 \%)\end{array}$ & $\begin{array}{l}0.77(0.48 \\
1.24)\end{array}$ & $1.04(0.59,1.82)$ \\
\hline$>4$ & $\begin{array}{l}49 \\
(10.9 \%)\end{array}$ & $\begin{array}{l}400 \\
(89.1 \%)\end{array}$ & $\begin{array}{l}0.90(0.53, \\
1.61)\end{array}$ & $1.17(0.59,2.33)$ \\
\hline \multicolumn{5}{|c|}{ Sources of drinking water } \\
\hline Improved & $\begin{array}{l}36 \\
(10.3 \%)\end{array}$ & $\begin{array}{l}314 \\
(89.7 \%)\end{array}$ & 1 & 1 \\
\hline Unimproved & $59(9.5 \%)$ & $\begin{array}{l}565 \\
(90.5 \%)\end{array}$ & $\begin{array}{l}1.10(0.71 \\
1.70)\end{array}$ & $0.96(0.57,1.61)$ \\
\hline
\end{tabular}

\section{Associations Between Children's Anemia And Malnutrition}

This study showed that among stunting, underweight, and wasting children, $41.5 \%, 45.8 \%$, and $35.0 \%$ were anemic respectively. Moreover, the percentages of stunting, underweighting and wasting were lower among anemic children as compared to no-anemic children. Stunting children were 1.31 times more likely to be anemic compared to those of not stunting (AOR $=1.31 ; 95 \% \mathrm{Cl} 0.94,1.82)$. Underweight children were 0.36 times less likely to be anemic compared to those of not underweight (AOR $=0.64 ; 95 \% \mathrm{Cl} 0.44$, 0.93). Wasting children were 1.80 times more likely to be anemic compared to those of not wasting (AOR $=1.80,95 \% \mathrm{Cl} 1.07,3.04)$. 
Table 4

Malnutrition associated with anemia among under-five children in Amhara Region, Ethiopia, EDHS 2016

\begin{tabular}{|c|c|c|c|c|c|}
\hline Variables & Categories & $\mathrm{n}$ & Anemic & P-value & AOR $(95 \% \mathrm{Cl})$ \\
\hline \multirow[t]{2}{*}{ Stunting } & Stunting & 452 & $146(41.5 \%)$ & 0.10 & $1.31(0.94,1.82)$ \\
\hline & Non stunting & 522 & $186(43.5 \%)$ & & 1 \\
\hline \multirow[t]{2}{*}{ Underweight } & Underweight & 327 & $104(45.8 \%)$ & 0.02 & $0.64(0.44,0.93)$ \\
\hline & Non underweight & 647 & $228(41.2 \%)$ & & 1 \\
\hline \multirow[t]{2}{*}{ Wasting } & Wasting & 180 & $28(35.0 \%)$ & 0.03 & $1.80(1.07,3.04)$ \\
\hline & Non wasting & 794 & 304 (43.4\%) & & 1 \\
\hline
\end{tabular}

\section{Discussion}

In this study, the prevalence of malnutrition and associated factors in Amhara region was assessed. The prevalence of stunting, underweight and wasting in Amhara region was $46.3 \%, 28.4 \%$ and $9.8 \%$, respectively. In this study, stunting and underweight are higher than that of the studies conducted in Ethiopia which were 38.3\% and 23.3\% [8], in Dale district 25.6\% and 19\% [15], Takusa district 36.5\% and $19.5 \%$ [16], respectively. The prevalence of stunting and underweight in this study is higher than the finding reported in Nairobi Peri-Urban Slum 30.2\% and 14.9\% [17], respectively. This could be due to there is a difference in obstacles to under-nutrition such as cultural differences and other socio-demographic characteristics. The prevalence of wasting in this study is lower compared to the study conducted in Ethiopia 10.1\% [8], in Haramaya district 10.7\% [14], in Dale Woreda 14\% [15], in Pakistan 10.7\% [18] and Kilimanjaro Region, Tanzania 24.7\% [5]. The prevalence reported in this study is higher compared to the one reported by Nairobi Peri-Urban slum 4.5\% [17]. This divergence might be due to the difference in socioeconomic background, variation in sample birth weight, dietary habits and type of meals among the study population.

About $23.1 \%$ of children were both stunting and underweight, $7.3 \%$ were both underweight and wasting, and only $4.5 \%$ of children had all the three conditions. The prevalence of both stunting and underweight at this study is higher than compared to the study conducted in Ethiopia 19.47\% [8] but lower than the study conducted in Kilimanjaro Region, Tanzania 33\% [5]. The prevalence of all the three conditions at this finding is lower than the study conducted in Kilimanjaro Region, Tanzania 12\% [5], but higher than in Ethiopia 3.87\% [8]. The variation might be due to socioeconomic background, geographical characteristics of the study area, access to health care, cultural difference in dietary habits and care practices. 
Among the factors considered in this study, Age of child in months, birth weight of the child at birth, mother highest education level, sex of household head and sources of drinking water were associated with stunting. The log odds of stunting were higher among children in the age group of 25-47 months and 48-59 months respectively as compared to the age group of $0-24$ months. This finding is in line with the studies conducted in Ethiopia [8], in Haramaya district [14], in Pakistan [18] and Kilimanjaro Region, Tanzania [5]. Compared to children $>4 \mathrm{~kg}$ birth weight at birth, the odds of stunting among children in the $2.5-4 \mathrm{~kg}$ birth weight was 0.01 times lower. The odds of stunting among children in the < $2.5 \mathrm{~kg}$ birth weight at birth were 1.60 times higher compared to children's $>4 \mathrm{~kg}$ birth weight at birth. This finding is supported by a study conducted previously in SNNPR, Ethiopia. [19].

The risk of stunting among children whose mothers attended secondary education was 0.70 times less compared to children whose mothers did not attend education. This finding is consistent with the study conducted in Bangladesh [20] and in Pakistan [18]. This is because if the level of education of the mother is low, her decision making and her contribution to the total family income will be low. This leads to the children stunting. The risk of stunting among children whose father household head was 0.49 times less compared to children whose mother household headed. Children from households that used unimproved drinking water were 1.47 times more likely to be at risk of stunting than children from households that used improved water. This finding is supported by the result of similar studies conducted in Haramaya District, Eastern Ethiopia [14].

The birth weight of the child at birth was associated with under-weight $(P<0.05)$. The risk of being underweight was 1.36 times more likely among children that were aged 48-59 months than those aged 0-24 months. This finding is supported by the study conducted in Ethiopia [8]. The risk of being underweight for children whose mother attended primary and secondary education were 0.23 and 0.35 times lower than children whose mothers who did not attend formal education respectively. This finding is supported by the study conducted in Ethiopia [8] and in Pakistan [18]. The sources of discrepancy might be due to maternal education contributes to proper infant feeding practices. Educated mothers might also have better income.

Children from a household with rich economic status were 0.09 times less likely to be under-weight compared to children living in a household with poor household economic status. This finding is supported by the study conducted in Ethiopia [8] and in Pakistan [18]. Children from rural areas were 1.16 times more likely to be underweight compared to children from urban areas. This finding is in agreement with the study conducted in Takusa district, Northwest Ethiopia [16]. The remarkable difference in the rate of underweight among rural and urban children might be differences in living circumstances, deviations in early screening of mothers at child conception in urban areas compared with rural areas, exposure to poor dietary diversity and greater risks of infections among rural children. Female children were 1.06 times more likely to be under-weighted as compared to male children. This study is against the studies conducted in Ethiopia [8], in Pakistan [18], in Bule Hora district, South Ethiopia [21] and in Dale Woreda, southern Ethiopia [15]. Children who were born with a $<2.5 \mathrm{~kg}$ birth weight were 1.80 times more likely to be under-weighted than children born $>4 \mathrm{~kg}$ birth weight and children who had born with $2.5-4 \mathrm{~kg}$ birth 
weight were 1.56 times more likely to be underweight than children born $>4 \mathrm{~kg}$ birth weight. This finding is in agreement with the study conducted in Ethiopia [8] and in Dale Woreda, southern Ethiopia [15].

Results of the multivariable binary logistic regression model showed that the type of toilet facility and sex of household head were significantly associated with wasting. Children living in a household with improved toilet type were 0.48 less likely to be wasting compared to children living in a household with unimproved toilet type. This finding is in agreement with finding in Bule Hora district, South Ethiopia [21]. Children from a male household head were 1.99 times higher compared to children from a female household head. Children of the rich household were 0.32 times less likely to be wasting compared to children living in a household with poor household economic status. This finding is supported by the study conducted in Ethiopia [8] and in Pakistan [18].

The risk of wasting was 1.08 and 1.52 times higher among children of $25-47$ and $48-59$ months than those 0-24 months, respectively. This finding is supported by the studies conducted in Dale Woreda, southern Ethiopia [15] and Kilimanjaro Region, Tanzania [5]. The odds of being wasting of children from rural areas were 1.18 times higher compared to children from urban areas. The variation might be due to food preference, food consumption patterns and inequalities in dietary diversity between urban and rural areas. This finding is consistent with the study conducted in Haramaya District, Eastern Ethiopia [14]. The odds of wasting were 0.04 times lower among female children than male children. This study is in line with the studies conducted in Bule Hora district, South Ethiopia [21], in Dale Woreda, southern Ethiopia [15], in Kilimanjaro Region, Tanzania [5] and Pakistan [18]. The odds of wasting was 1.17 times higher among children who lived in household members of $>4$ children who had lived in household members of 1-2. This finding is in line with the study conducted in Dale Woreda, southern Ethiopia [15]. The probable reason is that when there are too many children who are living together in the family, there may be a tendency for under-nutrition to occur.

This study showed that among stunting, underweight, and wasting children, $41.5 \%, 45.8 \%$, and $35.0 \%$ were anemic respectively. These findings are lower than compared with the study conducted in Ethiopia $61 \%, 64.3 \%$ and $68.2 \%$ respectively [8]. Moreover, the percentages of stunting, underweighting and wasting were lower among anemic children as compared to no-anemic children. Stunting children were 1.31 times more likely to be anemic compared to those of not stunting. Underweight children were 0.36 times less likely to be anemic compared to those of not underweight. Wasting children were 1.80 times more likely to be anemic compared to those of not wasting. These findings are supported by the study conducted in Ethiopia [8]. In the current study, anemia and malnutrition of children were highly associated with that anemic children were more likely to be malnutrition as compared to non-anemic [22].

\section{Conclusion}

This study showed individual- and community-level factors determined childhood malnutrition in Amhara region children. Among the factors considered in this study, Age of child in months, birth weight of the child at birth, mother highest education level, sex of household head, sources of drinking water and type 
of toilet facility were significantly associated with malnutrition in the Amhara region. Being underweight and wasted of children was significantly contributing to developing anemia. This finding would be directing the authors that malnutrition among under-five children was one of the public health problems in the Amhara region. The influence of these factors should be considered to develop strategies for reducing malnutrition in Amhara Region. Improving the living standards of children is important to get better health care, reduce child malnutrition, and child mortality. Finally, further researches on under-five children are recommended to investigate additional malnutrition factors.

\section{Limitations Of The Study}

A limitation was the use of a cross-sectional study design which could only generate a hypothesis regarding the role of independent variables on the nutritional status of children but not their cause and effect relationships.

\section{Abbreviations}

AOR

adjusted odds ratio; BMl:body mass index; COR:crude odds ratio; CSA:Central Statistical Agency; SNNPR:South Nations, Nationalities, and People Region; SPSS:Statistical Package for Social Science; DHS:Demographic and Health Surveys; EDHS:Ethiopian Demographic and Health Survey.

\section{Declarations}

\section{Acknowledgments}

The authors would like to thank Ethiopia Central Statistical Agency for permitting us to use the data for our study.

\section{Authors' contributions}

DK conceived the idea, drafts the manuscript and interpreted the results. DK and YM performed statistical analysis and help in results interpretation and writing. DK and YM critically reviewed the manuscript.

\section{Funding}

No funding was obtained for this study.

\section{Availability of data and materials}

The data set used and analyzed during the current study is available from the corresponding author on reasonable request (in SPSS code).

\section{Ethics approval and consent to participate}


Ethics approval and consent to participate The EDHS 2016 have taken into account the standard ethical

guidelines of the measure DHS program. The authors have obtained the data from measure DHS website (https://www.dhsprogram.com/data/dataset_admin/index.cfm) following their data obtaining procedure. The formal ethical clearance was obtained from the Demographic and Health Surveys (DHS) program.

\section{Consent for publication}

Not applicable.

\section{Competing interests}

The authors declare that they have no competing interests.

\section{References}

1. Antwi S. Malnutrition: missed opportunities for diagnosis. Ghana medical journal, 2008. 42(3).

2. Pelletier DL, Frongillo EA. Changes in child survival are strongly associated with changes in malnutrition in developing countries. J Nutr. 2003;133(1):107-19.

3. De Onis M, Blössner M. The World Health Organization global database on child growth and malnutrition: methodology and applications. Int J Epidemiol. 2003;32(4):518-26.

4. Icf C. Ethiopian demographic health survey 2011. Addis Ababa and Calverton: Central Statistical Agency (Ethiopia) and ICF International, 2012: p. 17-27.

5. Mgongo $\mathrm{M}$, et al. Underweight, stunting and wasting among children in Kilimanjaro Region, Tanzania; a population-based cross-sectional study. Int J Environ Res Public Health. 2017;14(5):509.

6. Debela BL, Shively G, Holden ST. Does Ethiopia's productive safety net program improve child nutrition? Food Security. 2015;7(6):1273-89.

7. Population EOot, Commission HC, Summary and statistical report of the 2007 population and housing census: population birth weight by age and sex. 2008: Federal Democratic Republic of Ethiopia, Population Census Commission.

8. Tekile AK, Woya AA, Basha GW. Prevalence of malnutrition and associated factors among under-five children in Ethiopia: evidence from the 2016 Ethiopia Demographic and Health Survey. BMC Res Notes. 2019;12(1):391.

9. Tariq J, et al. Factors associated with undernutrition in children under the age of two years: secondary data analysis based on the Pakistan demographic and health survey 2012-2013. Nutrients. 2018;10(6):676.

10. Talukder A. Factors associated with malnutrition among under-five children: illustration using Bangladesh demographic and health survey, 2014 data. Children. 2017;4(10):88.

11. Asim M, Nawaz Y. Child malnutrition in Pakistan: evidence from literature. Children. 2018;5(5):60.

12. Das S, Gulshan J. Different forms of malnutrition among under five children in Bangladesh: a cross sectional study on prevalence and determinants. BMC Nutrition. 2017;3(1):1. 
13. Akombi BJ, et al. Multilevel analysis of factors associated with wasting and underweight among children under-five years in Nigeria. Nutrients. 2017;9(1):44.

14. Yisak $\mathrm{H}$, Gobena T, Mesfin F. Prevalence and risk factors for under nutrition among children under five at Haramaya district, Eastern Ethiopia. BMC Pediatr. 2015;15(1):212.

15. Wolde $\mathrm{M}$, Berhan $\mathrm{Y}$, Chala $\mathrm{A}$. Determinants of underweight, stunting and wasting among schoolchildren. BMC Public Health. 2015;15(1):8.

16. Nigatu G, et al. Prevalence and associated factors of underweight among children 6-59 months of age in Takusa district, Northwest Ethiopia. Int J Equity Health. 2018;17(1):106.

17. Chesire $E$, et al. Determinants of under nutrition among school age children in a Nairobi peri-urban slum. East Afr Med J. 2008;85(10):471-9.

18. Khan S, Zaheer S, Safdar NF. Determinants of stunting, underweight and wasting among children $<5$ years of age: evidence from 2012-2013 Pakistan demographic and health survey. BMC Public Health. 2019;19(1):358.

19. Ayalew E, The Prevalence of Stunting and Associated Factors Among Children Age 6-59 Months At Mizan-Aman Town, Bench Maji Zone, Snnpr Region, Ethiopia, 2015, 2015, Addis Ababa University.

20. Siddiqi MNA, Haque MN, Goni MA. Malnutrition of under-five children: evidence from Bangladesh. Asian Journal of medical sciences. 2011;2(2):113-9.

21. Asfaw $M$, et al. Prevalence of undernutrition and associated factors among children aged between six to fifty nine months in Bule Hora district, South Ethiopia. BMC Public health. 2015;15(1):41.

22. Roba KT, et al. Anemia and undernutrition among children aged 6-23 months in two agroecological zones of rural Ethiopia. Pediatric health medicine therapeutics. 2016;7:131. 